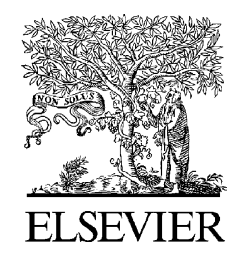

\title{
EDITORIAL
}

\section{Home oxygen therapy: the Canadian perspective}

It is very encouraging to learn of a health service making a concerted effort to improve its clinical effectiveness, as reported by Pearce in this issue of the Primary Care Respiratory Journal [1], especially when a new system follows reports authored by pre-eminent healthcare professionals interested in their patients receiving the maximum benefit from long-term oxygen therapy (LTOT) [2]. Since there has been a longstanding issue in the UK regarding the limitations of providing ambulatory oxygen, it would seem that at least one important comporsent of chronic disease management will be cesolved as from February 1st 2006 . Ha inguvorned through this issue in the Conaliang healthcare svstem y hith is similar tc the UK National Heing Service (NHS) insofar as the vast majority of patients are provided with a service that is virtually free of charge, my excitement is tempered by concerns regarding the issue of LTOT that are both administrative and clinical.

From February 1st 2006 in the UK, the provision of an integrated oxygen supply route provided by a single contractor within each new oxygen service region sounds attractive. However, despite the successful companies having been selected fairly, in fact, a series of regional monopolies have been created. During competitive bidding it is only natural to maximize the service claims and minimize the costs. What will happen should these services fall short after alternative options are no longer viable? It is in the interests of everyone that appropriate independent monitoring be part of the service provision, otherwise the new system risks shortfalls in equipment or timing that will be detrimental to patients. Perhaps the local evaluation teams who nominated their preferred providers should be given an oversight role? It would be as well to consider this issue as the new system starts.

Information gathering is another important aspect of an effective service. In the absence of clear demographic, clinical and administrative data, it will be almost impossible to make accurate cost predictions of the new system. A detailed, centralised indedenden database will enable many linportain questions related to cost and Certectiveness to be answered. An economic analysis, cpmptas the new system against the old, woulcertainly be helpful. The personnel and the consulting healthcare professionals responsible for information services should be identified promptly to the general practice community, in order for an open two-way communication to occur between the monitoring teams and the physicians using the new service.

In our experience in Canada, the issue of 'discussions' between the prescriber and the supplier can come to mean much more. Whereas in principle 'discussion' is always healthy, when financial implications are involved there may be circumstances in which the provider might encourage the clinician to prescribe equipment based on factors other than the best interests of the patient. Therefore, ascertaining who meets funding criteria, patient testing or re-evaluation, equipment recommendations, and questions regarding prescribing flow rates, should be strictly within the clinical domain - whether through the general practitioner's (GP's) office or, perhaps more easily, through a specific oxygen clinic.

In addition to the administrative issues, clinical issues abound. In my view these are more 
difficult to answer but they present interesting opportunities for clinical research, provided the new service attaches some funding for this purpose. Extending conclusions from the landmark trials of LTOT - in which it was found to be life extending for stable COPD patients with resting hypoxaemia $[3,4]$ - to other diagnostic groups and other circumstances such as transient hypoxaemia associated with sleep or exercise, or intermittent activity-related dyspnoea, moves from an evidence-based practice to a speculative one. The addition of ambulatory oxygen, though in many ways intuitive, is not yet strongly supported by the small amount of available data. Two well-designed trials $[5,6]$ reported very limited benefits from the provision of a portable system. Although some highly mobile patients will benefit from a portable system, many other patients will not. Therefore, appropriate testing, followup and supervision of patients' use of ambulatory oxygen is important if the new system is to be cost effective. Similarly, short-burst oxygen for dyspnoea, although popular with patients and some clinicians, is not strongly supported by evidence from prospective randomised controlled trials.

One way to increase the likelihood of LTOT being provided for those who could benefit from it, is to ensure a reasonable level of knowledes an ong prescribers. Educational suppor odes not appear to have been men $\rightarrow$ ond $\in$ d if the UK Departncent of Health's annúncement, despite its importance to, and implications for, many GPs and specialists. People learn in different ways, but one option is through the provision of accessible on-line information at no cost. In Canada we addressed this issue with a group of clinicians interested in the subject, who authored an interactive learning module to address the indications, prescribing options, and evidence in support of, LTOT. The group included GPs, specialists, nurses and respiratory therapists (not yet available in the UK). The module was extensively peerreviewed both nationally and internationally. It was endorsed by the Canadian College of Family Physicians, the Canadian Thoracic Society and the American College of Chest Physicians. In response to the user's requests, a patient companion module - ideal for self-help groups or physicians' offices - was also developed. This was endorsed by the most prominent organizations responsible for patient education; the Canadian Lung Association and the Ontario Ministry of Health. The beauty of on-line learning is that its use can be tracked and its contents can be updated promptly as new information becomes available.
The modules have been available for some time in Canada, the US, Australia, New Zealand and South Africa.

In response to a request by several of the UK contractors these on-line learning modules have been customized for the UK with the assistance of a national medical advisory panel. The sponsoring companies, whose logos appear at the beginning and the end of the modules, had no role in the reviewer selection or the module content. They provided an unrestricted educational grant in order for the information to be peer-reviewed, modified and then hosted on the site of "DiscoveryCampus" for a three-year period, free of charge to any healthcare professional or patient. The clinician module has been endorsed by the UK General Practice Airways Group (GPIAG), the International Primary Care Respiratory Group (IPCRG) as well as the UK National Respiratory Training Centre (NRTC). The British Lung Foundation (BLF) has agreed to review and provide input to the patient module. These programmes will launch within the next few days. The clinician module can be accessed by registering on the DiscoveryCampus site, as shown below. The patient module can be accessed immediatety by clicking on the site or on CD thrpugh al sponsoring contractor. Such an td privach will reach many GPs and patient users. Hopeflity', Aase of this on-line learning tool villad(d) in a small way to the effectiveness of the new system for home oxygen provision coming into force in the UK as from February 1st 2006.

Access to these modules is as follows:

UK LTOT Clinician Module

http:/ /www.discoverycampus.com

register and login in to course: RSP001UK

UK LTOT Patient Module

http://www.discoverycampus.com/ pub010/pub010.htm

\section{References}

[1] Pearce L. Changes to the home oxygen provision service in the UK. Prim Care Resp J 2005;14(6):279-81.

[2] Rudolf $M$, Wedzicha W, Calverley $P$ et al. Domiciliary oxygen therapy services. Clinical Guidelines and advice for prescribers. A report of the Royal College of Physicians. 1999. London, Royal College of Physicians. ISBN 186016 0816.

[3] Continuous or nocturnal oxygen therapy in hypoxemic chronic obstructive lung disease. A clinical trial. Nocturnal Oxygen Therapy Trial Group (NOTT). Ann Int Med 1980;93:391-98. 
[4] Report of the British Medical Research Council Working Party: Long term domiciliary oxygen therapy in chronic hypoxic cor pulmonale complicating chronic bronchitis and emphysema. Lancet 1981;1:681-86.

[5] Vergeret J, Brambilla C, Mounier L. Portable oxygen therapy: use and benefit in hypoxaemic COPD patients on long-term oxygen therapy. ERJ 1989;2:20-5.

[6] Lacasse Y, Lecours R, Pelletier C, Begin R, Maltais F. Randomised trial of ambulatory oxygen in oxygen-dependent COPD. ERJ 2005;25(6):1032-8.
Roger S. Goldstein

(Professor of Medicine \& Physical Therapy)* University of Toronto, West Park Healthcare Centre, 82 Buttonwood Avenue, Toronto,

Ontario, Canada M6M 2J5

*Tel.: +1 416243 3631; fax: +1 4162438947.

E-mail address: rgoldstein@westpark.org

10 May 2005 